\title{
Mitochondrial A12308G polymorphism affects clinical features in patients with single mtDNA macrodeletion
}

\author{
Marco Crimi ${ }^{*}{ }^{1}$, Roberto Del Bo ${ }^{1}$, Sara Galbiati ${ }^{1}$, Monica Sciacco ${ }^{1}$, Andreina Bordoni ${ }^{1}$, \\ Nereo Bresolin ${ }^{1,2}$ and Giacomo Pietro Comi ${ }^{1}$
}

${ }^{1}$ Centro Dino Ferrari, Centro di eccellenza per le malattie neuro-degenerative, Dipartimento di Scienze Neurologiche, Università degli Studi di Milano, I.R.C.C.S. Ospedale Maggiore Policlinico, Milano, Italy; ${ }^{2}$ I.R.C.C.S. 'E. Medea', La Nostra Famiglia - Bosisio Parini (LC), Italy

\begin{abstract}
Mitochondrial (mt)DNA alterations cause cellular energy failure and respiratory chain dysfunction. Single large-scale rearrangements represent the most common mtDNA mutations and are responsible for very variable clinical manifestations. Here, we show an increased frequency of the A12308G substitution, a common polymorphism used to define the European mtDNA haplogroup $U$, in mitochondrial patients carrying mtDNA single macrodeletion. In this group of patients, A12308C substitution is associated with a higher relative risk of developing pigmentary retinal degeneration, short stature, dysphasia-dysarthria and cardiac conduction defects. MtDNA haplotype might modulate the clinical expression of mitochondrial encephalomyopathies due to mtDNA macrodeletions. European Journal of Human Genetics (2003) 11, 896-898. doi:10.1038/sj.ejhg.5201056
\end{abstract}

Keywords: mitochondrial DNA; macrodeletion; haplotype

\section{Introduction}

The human mitochondrial DNA (mtDNA) consists of circular double-stranded molecules 16569 base pairs in size that code for 37 genes. Mitochondrial genome is responsible for the intramitochondrial synthesis of 13 proteins, all involved in the respiratory chain. Molecular defects of mtDNA, therefore, cause cellular energy failure resulting from respiratory chain dysfunction. ${ }^{1}$ Mitochondrial disorders are human genetic diseases with extremely variable clinic and genetic features. Pathogenic mtDNA defects can be divided into two groups: large-scale rearrangements (from a few hundred base pairs to $>10 \mathrm{~kb}$ ) and point mutations affecting both proteinencoding genes and transfer and ribosomal RNA genes.

*Correspondence: Dr Marco Crimi, Dipartimento di Scienze Neurologiche (Pad.ne Ponti), via F. Sforza, no. 35, 20122 Milano, Italy.

Tel: + 39 255033843; Fax: + 39 250320430;

E-mail: neurogene@policlinico.mi.it

Received 11 October 2002; revised 28 April 2003; accepted 23 May 2003
MtDNA alterations may be maternally inherited or arise from de novo germ-line or somatic mutations. Single largescale rearrangements represent the most common mtDNA mutations and are most often found in chronic progressive external ophthalmoplegia and the Kearns Sayre syndrome. They occur only in sporadic cases, therefore hampering the study of the possible modulating role of autosomal genes. Clinical manifestations are very variable: the dominant clinical features include progressive eye signs (such as ptosis, restricted eye movements and pigmentary retinopathy) and hearing loss. Neurological symptoms include, muscle weakness, ataxia and cognitive impairment. Short stature and dysphasia-dysarthria are also common. Cardiac conduction defect, diabetes or other endocrine disorders and parkinsonism (rarely) ${ }^{2}$ may also be determined by mtDNA large deletion.

MtDNA has a very high mutation rate (10-20 times higher than nuclear DNA in humans); this property is used to define ethnic populations: several studies have revealed ancestral-associated polymorphisms whose combination 
defines different mtDNA subgroups (mtDNA haplogroups). One of these, the European haplogroup $U$ (comprising the subhaplogroups: U1-U6 and K) is defined by the A12308G polymorphism. Both the subhaplogroups $U$ and $K$ branched from the same multifurcation characterized by the A12308G state, but the relationship between RFLP clarifies that $\mathrm{K}$ is embedded within $\mathrm{U}$ through the variant A9052G. ${ }^{3}$ The 12308 site is situated in the tRNA ${ }^{\text {Leu (CUN) }}$ gene. The tRNA ${ }^{\text {Leu (CUN) }}$ encodes for the most represented aminoacid in the mt-respiratory chain, suggesting a key role of this tRNA in mtDNA-coded OXPHOS subunits.

\section{Materials and methods}

We investigated the A12308G and A9052G frequencies in 429 unrelated subjects referred to our Department of Neurology; already described procedures were followed. ${ }^{2,4}$ In all, 254 subjects, free of any neuro-muscular disorder, were considered as controls; 175 patients were diagnosed with a mitochondrial disorder and were classified according to their mtDNA molecular defects in three different subgroups: 57 single-point mutations (25 LHON, nine MELAS A3243G, five CPEO A3243G, two MERRF A8344G, one MIMyCa, one NARP and 14 as others rare heteroplasmic point mutations in coding regions); 55 muscle mtDNA multiple deletions (familial and sporadic cases) and 63 single macrodeletions (the 'common' deletion was the most represented): the heteroplasmic quote was muscleevaluated with the $\chi^{2}$ test as statistical approach (in all tests, a $P$-value below 0.05 was considered significant).

\section{Results}

We observed that the frequency of the A12308G transition was similar in controls (18.9\%), point mutation $(17.5 \%)$ and multiple deletions patients (18.2\%). Surprisingly, the frequency of the A12308G polymorphism increased to $38.1 \%(P<0.005)$ in the group of mitochondrial patients carrying single macrodeletions (Figure 1). To define the mtDNA subgroup within the $U$ haplogroup, we checked the 24 patients with single macrodeletions carrying the A12308G polymorphism for the A9052G variant. A9052G, which determines the subhaplogroup $\mathrm{K}$ in combination with $\mathrm{A} 12308 \mathrm{G}$, is present in 11 cases (45.8\%: a frequence higher than that observed in our healthy control population, assessed at 24.3\%). Consequently, the frequency of the other $\mathrm{U}$ subhaplogroups (not K) was lower than that found in healthy controls (54.2 versus $75.7 \%$ ).

To investigate whether A12308G may be relevant to the high clinical phenotypic diversity observed in these patients, we analyzed the association between clinical features in the 63 single-macrodeleted patients and the presence of this polymorphism. Patients with the A12308G transition had a higher relative risk of developing pigmentary retinopathy, short stature, dysphasia-dysarthria and

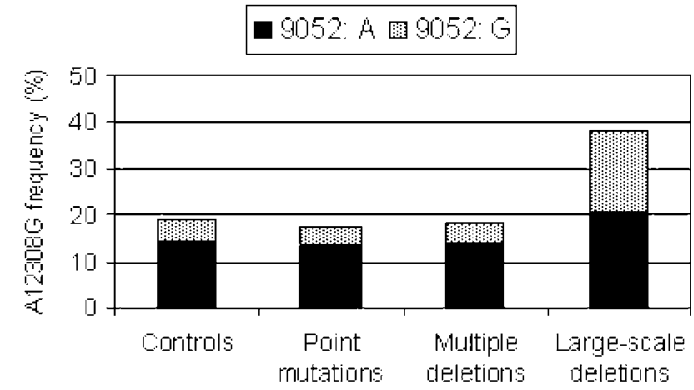

Figure 1 Comparison of the A12308G-polymorphism's frequency in control subjects and different mitochondrialpatient groups: the diagrams are divided according to the absence (full) or presence (dot) of A9052G polymorphism.

cardiac conduction defects $(P<0.005$, Table 1$)$. The two groups did not differ in terms of actual age (A12308: mean age: 42 years, SD: 16.5; G12308: 33.2 years, SD: 18.4$)$ and age of onset (A12308: mean age: 24.4 years, SD: 13.3; G12308: 20.8 years, SD: 12.9), although both ages were younger in G12308 single deleted patients. A9052G does not influence the clinical phenotypic diversity. Furthermore the Southern blot densitometric analysis of PvuIIdigested muscle patients' DNA revealed that the levels of heteroplasmic deletion (percentage of deleted mtDNA) did not significantly differ between the two groups (Table 1).

\section{Discussion}

The study of the 63 pathologically and genetically confirmed cases of single mtDNA macrodeletion revealed a certain association with mtDNA polymorphism A12308G when compared to control subjects or other mitochondrial mutations. Particularly, we observed a strong relationship with a mtDNA subhaplogroup, the haplotype K. Although there is no a priori reason why haplogroup $\mathrm{K}$ should be associated with the mtDNA macrodeletion, the most likely explanation is that this subhaplogroup could be a risk factor for mtDNA single macrodeletion. Subhaplogroup K is defined by the combination with two variants: A9052G and A12308G. The A12308G change is a well known polymorphism; ${ }^{4}$ although its biological significance remains to be evaluated, it has been described as a predictive factor for strokes in MELAS patients. ${ }^{5}$ Among the patients carrying the A3243G mutations, seven had recurrent stroke-like episodes, while two had only one cerebrovascular episode; three of those with multiple strokes also bear the A12308G haplotype. The other tRNA-mutation classes were too scanty to make meaningful comparisons.

A12308G has a synergistic effect with the mtDNA T12297C mutation associated with dilated cardiomyopathy. ${ }^{6}$ Besides, a convincing association has been proved between the aging process and mtDNA haplogroups. ${ }^{7,8}$ Our data suggest that the presence of the A12308G polymorph- 
Table 1 Frequency of clinical features in 63 patients with mtDNA's single large-scale rearrangements: the frequencies are stratified for the absence/presence of A12308G polymorphism

\begin{tabular}{|c|c|c|c|c|c|c|}
\hline & \multicolumn{3}{|c|}{ 12308: $A$} & \multicolumn{2}{|c|}{ 12308: G } & \multirow[b]{2}{*}{$P^{*}$} \\
\hline & Tot. & Cases & $\%$ & Cases & $\%$ & \\
\hline $\begin{array}{l}\text { All } \\
\text { Inclusion criteria }\end{array}$ & 63 & 39 & 61.9 & 24 & 38.1 & - \\
\hline Age of onset & 63 & 40 & $24.4(13.3)$ & 23 & $20.8(12.9)$ & NS \\
\hline Lactic acidemia** & 40 & 23 & $23.1(14.7)$ & 17 & $30.2(11.9)$ & NS \\
\hline Heteroplasmy & 63 & 40 & $45.9(21.6)$ & 23 & $46.4(19.1)$ & NS \\
\hline \multicolumn{7}{|l|}{ Clinical features } \\
\hline Cardiac conduction defects & 27 & 11 & 40.7 & 16 & 59.3 & $<0.005$ \\
\hline Pigmentary retinal degeneration & 19 & 7 & 36.8 & 12 & 63.2 & $<0.005$ \\
\hline Dysarthria/dysphasia & 16 & 6 & 37.5 & 10 & 62.5 & $<0.005$ \\
\hline Short stature & 10 & 3 & 30.0 & 7 & 70.0 & $<0.005$ \\
\hline Neuropathy & 6 & 4 & 66.7 & 2 & 33.3 & NS \\
\hline Cerebellar signs & 22 & 12 & 54.5 & 10 & 45.5 & NS \\
\hline Cognitive impairment & 6 & 4 & 66.7 & 2 & 33.3 & NS \\
\hline Hearing loss & 24 & 14 & 58.3 & 10 & 41.7 & NS \\
\hline Myopathy & 43 & 26 & 60.5 & 17 & 39.5 & NS \\
\hline Diabetes & 6 & 4 & 66.7 & 2 & 33.3 & NS \\
\hline Ophthalmoplegia & 52 & 33 & 63.5 & 19 & 36.5 & NS \\
\hline Ptosis & 58 & 36 & 62.1 & 22 & 37.9 & NS \\
\hline
\end{tabular}

${ }^{*} \chi^{2}$ test; ${ }^{* *}$ rest serum lactate level expressed as $\mathrm{mg} / \mathrm{dl}$; values are given as mean and Standard Deviation (SD).

ism may have a harmful effect on mtDNA when this is already damaged by heteroplasmic large deletions: the potential for the clinical manifestation is enhanced when the single macrodeletion occurs upon the A12308G background. Particularly, 27 out of 63 patients with single large scale rearrangement had cardiac conduction defects (16 with the 12308: G polymorphism) in this study. This may suggest that the A12308G polymorphism may also have an organ-specific effect outside the CNS. Several factors are likely to influence the clinical phenotype of mtDNArelated disorders, namely tissue segregation and amount of mutated DNA, tissue energetic needs and other genetic determinants, including mtDNA haplogroups. The experimental observations highlight the existence of commonly occurring clinical patterns: the identification of these factors might have relevant prognostic and genetic counseling implications.

\section{Acknowledgements}

Contract grant sponsor: the financial support of 'Associazione amici del Centro Dino Ferrari' is gratefully acknowledged. The Telethon 'Bank of DNA, nerve and muscle tissues' (GTF02008) was the source of the muscle for this study.

\section{References}

1 Wallace DC, Brown MD, Lott MT: Mitochondrial DNA variation in human evolution and disease. Gene 1999; 238: 211-230, Review.

2 Zeviani M, Moraes CT, DiMauro $\mathrm{S}$ et al: Deletions of mitochondrial DNA in Kearns-Sayre syndrome. Neurology 1998; 51: $1525-1532$.

3 Macaulay V, Richards M, Hickey E et al: The emerging tree of west Eurasian mtDNAs: a synthesis of control-region sequences and RFLPs. Am J Hum Genet 1999; 64: 232-249.

4 Sternberg D, Danan C, Lombes A et al: Exhaustive scanning approach to screen all the mitochondrial tRNA genes for mutations and its application to the investigation of 35 independent patients with mitochondrial disorders. Hum Mol Genet 1998; 7: 33-42.

5 Pulkes T, Sweeney MG, Hanna MG: Increased risk of stroke in patients with the A12308G polymorphism in mitochondria. Lancet 2000; 356: 2068-2069.

6 Grasso M, Diegoli M, Brega A, Campana C, Tavazzi L, Arbustini E: The mitochondrial DNA mutation T12297C affects a highly conserved nucleotide of tRNA(Leu(CUN)) and is associated with dilated cardiomyopathy. Eur J Hum Genet 2001; 9: 311-315.

7 Rose G, Passarino G, Carrieri G et al: Paradoxes in longevity: sequence analysis of mtDNA haplogroup $\mathrm{J}$ in centenarians. Eur J Hum Genet 2001; 9: 701-707.

8 De Benedictis G, Carrieri G, Varcasia O, Bonafe M, Francesci C: Inherited variability of the mitochondrial genome and successful aging in humans. Ann NY Acad Sci 2000; 908: 208-218, Review. 